\title{
Evaluation of Mast-ID 15 system for identification of fresh clinical isolates of Enterobacteriaceae and Acinetobacter
}

\author{
A P Shakespeare, G M Megson, P A Jones, D B Drucker, M G L Keaney, L A Ganguli
}

\begin{abstract}
Aims: To assess the accuracy of the Mast-ID 15 system compared with API 20 $E$ for the identification of stock and fresh clinical strains of Enterobacteriaceae and Acinetobacter spp; to compare the accuracy of 19 pin and 36 pin multipoint inoculator heads.

Methods: One hundred frozen stock cultures of Enterobacteriaceae and Acinetobacter spp which had previously been identified by the API 20E were classified by the Mast-ID using 19 and 36 pin multipoint inoculator heads. Reproducibility was determined by testing 36 randomly selected organisms in duplicate. Four hundred and sixty nine consecutive fresh clinical isolates of Enterobaccteriaceae and Acinetobacter spp were identified by the Mast-ID using a 36 pin multipoint inoculator and by the API 20E. Reproducibility for the fresh isolates was determined by testing 96 randomly selected strains in duplicate.

Results: The Mast-ID 15 identified 82\% and $85 \%$ of frozen strains to species level and reproducibility was $80 \%$ and $86 \%$ using 19 and 36 pin inoculator heads, respectively. Of the 469 fresh clinical isolates, the Mast-ID identified $70 \%$ of strains to species level; $19 \%$ were not identified and $11 \%$ were identified incorrectly by comparison with the API 20E. The Mast-ID achieved a reproducibility level of $80 \%$ with the fresh clinical isolates.
\end{abstract}

Conclusions: The use of a 36 pin multipoint inoculator head in preference to the standard 19 pin head for the Mast-ID was advantageous as it allowed greater numbers of strains to be identified at a reduced cost. Unfortunately, in our hands, the Mast-ID system was insufficiently accurate for routine use in the clinical laboratory. Modifications to some of the problematic tests may result in a sufficient increase in accuracy and reproducibility to make the system beneficial in the routine clinical laboratory.

Commercial kit systems for the identification of enterobacteria are easy to use, accurate, and reproducible. High costs, however, prohibit the identification of large numbers of organisms. Most laboratories are therefore restricted to identifying only small numbers of selected isolates. Systems which use a multipoint inoculator in conjunction with a series of biochemical tests incorporated into agar plates are now available. One such commercially available method is the Mast-ID 15 system for the identification of enterobacteria. This has been evaluated by Holmes and Dawson ${ }^{1}$ using 621 strains-both reference strains of the National Collection of Type Cultures (NCTC) and those referred to the NCTC for identification; $88 \%$ of strains were correctly identified, most to species level. Their study was performed using 19 pin multipoint inoculator heads, as recommended by the manufacturer. The Mast-ID system, if used in conjunction with established breakpoint sensitivity testing (using the same inocula), would be economical to use. Because this laboratory routinely uses a 36 pin multipoint inoculation system for breakpoint sensitivity testing, the first aim of the present study was to compare the accuracy and reproducibility of the Mast-ID 15 system using 36 pin and 19 pin multipoint inoculator heads. The system was then evaluated for identification of routinely isolated fresh clinical strains. The API 20E was used as the standard for reference because this was shown to be superior in terms of both accuracy and reproducibility to 10 commercial kits evaluated by the Welsh Standing Specialist Advisory Working Group in Microbiology. ${ }^{2}$

The use of API as a yardstick for speciation is widely accepted. Nevertheless, it is theoretically possible that a procedure under evaluation, such as the Mast ID, might correctly speciate an isolate and yet not be credited with a correct speciation due to comparison with an erroneous API result.

\section{Methods}

To compare results obtained with the Mast-ID 15 system used in conjunction with 36 pin and 19 pin multipoint inoculator heads, 100 frozen laboratory stock cultures were tested. These had previously been identified using the API $20 \mathrm{E}$, all with a relative likelihood of identification of at least $0 \cdot 800,{ }^{1}$ and all were subcultured twice on cysteine lactose electrolyte-deficient agar (CLED; LAB-M) before testing. Reproducibility was subsequently determined by testing 36 randomly selected organisms in duplicate with both inoculator heads.

Consecutive fresh clinical isolates of aerobic Gram negative rods were classificed by the API 
Table 1 Identification of stock cultures of Enterobacteriaceae and Acinetobacter to species level by Mast-ID 15 comparing 19 pin and 36 pin multipoint inoculators

\begin{tabular}{lccc}
\hline & & \multicolumn{2}{c}{$\begin{array}{c}\text { No of species identified } \\
\text { to species level` }\end{array}$} \\
\cline { 2 - 4 } Organism & No of strains & 19 pin & 36 pin \\
\hline Acinetobacter spp $\dagger$ & 8 & 8 & 8 \\
Aeromonas hydrophila & 2 & 1 & 1 \\
Citrobacter diversus & 1 & 1 & 1 \\
Citrobacter freundii & 1 & 1 & 1 \\
Escherichia coli & 24 & 18 & 3 \\
Enterobacter aerogenes & 3 & 7 & 8 \\
Enterobacter cloacae & 8 & 5 & 5 \\
Klebsiella oxytoca & 6 & 5 & 5 \\
Klebsiella pneumoniae/aerogenes & 5 & 0 & 0 \\
Klebsiella rhinoscleromatis & 1 & 5 & 5 \\
Morganella morganii & 5 & 17 & 17 \\
Proteus mirabilis & 18 & 2 & 2 \\
Proteus vulgaris & 2 & 0 & 0 \\
Providencia stuartii & 2 & 1 & 1 \\
Salmonella sp & 1 & 1 & 1 \\
Serratia liquefaciens & 3 & 6 & 7 \\
Serratia marcescens & 8 & 0 & 1 \\
Shigella sonnei & 1 & 1 & 0 \\
Yersinia enterocolitica & 1 & 82 & 85 \\
Total & 100 & 7 & \\
\hline
\end{tabular}

ॠRelative likelihood of identification of $>0.800$ using API $20 \mathrm{E}$ as the reference standard for correct identification.

+ Acinetobacter spp identified to genus level only using API $20 \mathrm{E}$.

$20 \mathrm{E}$, of which 469 had a relative likelihood of identification of at least $0 \cdot 800^{1}$. These 469 organisms were then identified using the MastID system and a 36 pin inoculator. All isolates were from blood cultures, soft tissue and wounds, sputum and urine. Reproducibility of the system for fresh clinical isolates was then ascertained by testing 96 randomly selected strains in duplicate.

\section{BIOCHEMICAL TESTS}

Fourteen biochemical test agars (Mast)namely amygdalin, inositol, melibiose, rhamnose, sorbitol, sucrose, dulcitol, xylose, malonate, ortho-nitro-phenyl-galactoside (ONPG), phenylalanine, urea, ornithine decarboxylase, indole and motility test agar-were prepared according to the manufacturer's instructions. All were dispensed into $9 \mathrm{~cm}$ Petri dishes except motility test agar which was distributed in $4 \mathrm{ml}$ volumes into the chambers of square "repli dishes" (Sterilin) divided into 25 equal compartments. Carbohydrate agar base (Mast) was included as a supplementary test for use as a negative control for interpreting carbo-

Table 2 Identification of fresh clinical isolates of Enterobacteriaceae and Acinetobacter to species level by Mast-ID 15 system using 36 pin multipoint inoculator

\begin{tabular}{|c|c|c|c|}
\hline \multirow[b]{2}{*}{$\begin{array}{l}\text { Organism } \\
\text { Acinetobacter spp } \dagger \\
\text { Citrobacter freundii } \\
\text { Enterobacter aerogenes } \\
\text { Enterobacter agglomerans } \\
\text { Enterobacter cloacae } \\
\text { Escherichia coli } \\
\text { Klebsiella oxytoca } \\
\text { Klebsiella pneumoniae/aerogenes } \\
\text { Morganella morganii } \\
\text { Proteus mirabilis } \\
\text { Proteus vulgaris } \\
\text { Providencia stuartii } \\
\text { Salmonella sp } \\
\text { Serratia liquefaciens } \\
\text { Serratia marcescens }\end{array}$} & \multirow[b]{2}{*}{$\begin{array}{r}\text { No of strains tested } \\
14 \\
13 \\
7 \\
4 \\
38 \\
191 \\
27 \\
39 \\
13 \\
93 \\
7 \\
7 \\
1 \\
4 \\
11\end{array}$} & \multicolumn{2}{|c|}{$\begin{array}{l}\text { No of strains } \\
\text { identified correctly } y^{\star}(\%)\end{array}$} \\
\hline & & $\begin{array}{r}6 \\
7 \\
4 \\
1 \\
20 \\
147 \\
16 \\
9 \\
10 \\
87 \\
6 \\
2 \\
1 \\
2 \\
9\end{array}$ & $\begin{array}{l}(43) \\
(54) \\
(57) \\
(25) \\
(53) \\
(77) \\
(59) \\
(23) \\
(77) \\
(93) \\
(86) \\
(29) \\
(100) \\
(50) \\
(82)\end{array}$ \\
\hline Total & 469 & 327 & (70) \\
\hline
\end{tabular}

$\star$ Relative likelihood of identification of $>0.800$ using API $20 \mathrm{E}$ as the reference standard for correct identification.

$\dagger$ Acinetobacter spp identified to genus level only using API $20 \mathrm{E}$. hydrate reactions. The inoculum for all tests was a four hour culture in Iso-sensitest broth (Oxoid) which was also subcultured on to CLED to check purity. The media were inoculated with a 19 pin or 36 pin inoculator as required, except for the motility test agar squares which were stab inoculated from broth suspensions with a straight wire in the centre of the chambers (one strain per chamber). Once the inoculum spots had dried those on the malonate and urea plates were isolated by cutting through the agar with a sterile scalpel, thus helping to prevent diffusion of colour change from positive colonies. All tests were incubated at $37^{\circ} \mathrm{C}$ for 18 hours and after incubation the indole plate was flooded with 4dimethylamino-cinnamaldehyde (DMCA). All results were interpreted visually with reference to the descriptions supplied by Mast.

The results of the 15 tests were used to generate a five digit profile number for each organism which was then compared with those in the profile index book "Mast-ID Database" (Issue No 1, July 1986).

\section{IDENTIFICATION}

As with the API $20 \mathrm{E}$ system, an isolate was considered to have been identified by the MastID 15 system only if the relative likelihood of identification had a minimum value of $0 \cdot 800^{1}$.

\section{Results}

A comparison of the results obtained with the two multipoint inoculator heads (table 1) shows that the Mast-ID 15 system identifies $82 \%$ and $85 \%$ of stock strains with the 19 pin and 36 pin heads, respectively. The level of reproducibility attained using the 36 pin inoculator $(86 \%)$ was again slightly better than that with the 19 pin inoculator $(80 \%)$.

Four hundred and sixty nine fresh clinical isolates were tested using the Mast-ID 15 system in conjunction with a 36 pin inoculator. The Mast-ID 15 system identified $70 \%$ of isolates to species level (table 2): $19 \%$ were not identified-that is, their profile number was not listed in the profile index book, or the relative likelihood of identification at species level was $<0.800$ - and $11 \%$ were identified incorrectly. Figures for generic identification of isolates are listed in table $3 ; 78 \%$ of strains were identified to genus level, $13 \%$ were not identified, and $9 \%$ were identified incorrectly. The Mast-ID 15 system attained an $80 \%$ level of reproducibility when identifying fresh clinical isolates. Certain tests were repeatedly difficult to interpret irrespective of the organism tested. In particular, the indole, malonate, ONPG, urea and motility tests were difficult to interpret and contributed to misidentification and poor reproducibility.

\section{Discussion}

The comparison of 36 pin and 19 pin inoculator heads (table 1) indicated that increasing the number of inoculation sites per plate did not impair the accuracy of the Mast-ID 15 system when identifying stock cultures of Enterobacteriaceae and Acinetobacter spp.

Diffusion colour changes from positive 
Table 3 Identification of fresh clinical isolates of Enterobacteriaceae and Acinetobacter to genus level by Mast-ID 15 system using 36 pin multipoint inoculator

\begin{tabular}{|c|c|c|c|}
\hline \multirow[t]{2}{*}{ Organism } & \multirow[t]{2}{*}{ No of strains tested } & \multicolumn{2}{|c|}{$\begin{array}{l}\text { No of strains } \\
\text { identified correctly }{ }^{\star}(\%)\end{array}$} \\
\hline & & & $(\%)$ \\
\hline $\begin{array}{l}\text { Acinetobacter spp } \\
\text { Citrobacter spp } \\
\text { Enterobacter spp } \\
\text { Escherichia coli } \\
\text { Klebsiella spp } \\
\text { Morganella spp } \\
\text { Proteus spp } \\
\text { Providencia spp } \\
\text { Salmonella sp } \\
\text { Serratia spp }\end{array}$ & $\begin{array}{r}14 \\
13 \\
49 \\
191 \\
66 \\
13 \\
100 \\
7 \\
1 \\
15\end{array}$ & $\begin{array}{r}6 \\
7 \\
29 \\
147 \\
60 \\
10 \\
93 \\
4 \\
1 \\
11\end{array}$ & $\begin{array}{l}(43) \\
(54) \\
(59) \\
(77) \\
(91) \\
(77) \\
(93) \\
(57) \\
(100) \\
(81)\end{array}$ \\
\hline Total & 469 & 368 & (78) \\
\hline
\end{tabular}

$\star$ Relative likelihood of identification of $>0.800$ using API 20E as the reference standard for correct identification. would have been of value as it has a high discriminatory value for $E$ coli.

Members of the genus Proteus were acceptably identified by the Mast-ID. Fresh isolates of $P$ vulgaris and $P$ mirabilis were classified in $86 \%$ and $93 \%$ of cases, respectively.

Klebsiella isolates were accurately identified to genus level $(91 \%)$ but speciation was problematic (table 2 ). Difficulty with the urea and malonate tests was in part responsible for this. The inclusion of a lysine decarboxylase test would have been of use in the assignation of isolates to the genus and in their speciation. Problems speciating Enterobacter strains were also encountered, in particular the motility test was difficult to interpret $(75 \%$ of strains being reported as non-motile).

Some tests repeatedly proved difficult to interpret. Of particular note, the indole, malonate, ONPG, urea and motility tests were frequently the cause of identification failure, misidentification and reproducibility problems. Isolation of inoculum spots has been suggested in previous studies (Holmes and Dawson ${ }^{1}$; Pease ${ }^{5}$ ) to prevent colour diffusing from positive colonies and affecting neighbouring negative colonies. This was applied to the malonate and urea tests in this study and was helpful in some cases, but it could also have been used for the ONPG test where diffusion of colour from positive colonies was found to be a problem. Further reduction of colour diffusion on these agars by the addition of an extra $1 \%$ agar has been shown in our laboratory, but these results are not included in this study because they involve modifications to the standard Mast media and therefore would invalidate the use of the standard database.

In our hands the Mast-ID 15 system is not sufficiently accurate for the identification of fresh clinical isolates to either species or genus level. The system, if more accurate, would be advantageous in a routine hospital laboratory because it permits the testing of a much larger number of isolates at about $10 \%$ of the cost per isolate of the API $20 \mathrm{E}$ (based on the testing of 36 isolates at 1991 prices). The testing of large numbers of strains is also less time consuming than with the API $20 \mathrm{E}$. If combined with "breakpoint" sensitivity testing, the same inocula can also be used for both systems. necessarily the same selection of tests comprising the Mast-ID 15 system, and levels of identification ranged from $47 \%$ to $92 \%$ at species level and $69 \%$ to $95 \%$ at genus level.

A recent survey of 641 consecutive Enterobacteriaceae and Acinetobacter isolates in our laboratory showed that $84.2 \%$ of isolates were either Escherichia coli (39.8\%), Proteus spp. $(20.4 \%)$, Klebsiella spp $(12 \cdot 3 \%)$ or Enterobacter spp (11.7\%) (G Megson, unpublished data). It is essential that any identification system intended for use in the routine hospital laboratory should provide for the accurate identification and speciation of these genera.

When the Mast-ID was used to identify stock strains and clinical isolates of $E$ coli only $\mathbf{7 7 \%}$ of strains were correctly identified (tables 2 and 3). Problems with interpretation of the ONPG, indole, and malonate tests were seen to be responsible for some of the misidentifications. The inclusion of $\beta$-glucuronidase agar
We thank Dr Derek Law for reviewing the manuscript and Mrs Sue Ferendenus for help with the typing.

1 Holmes B, Dawson CA. Evaluation of Mast-ID 15 system and Acinetobacter. J Clin Pathol 1987;40:1168-73.

2 Bennett $\mathrm{CH}$, Joynson DH. Kit systems for identifying Gram-negative aerobic bacilli: report of the Welsh Standing Specialist Advisory Working Group in Microbiology. J Clin Pathol 1986;39:666-71.

3 Clayton P, Feltham RK, Mitchell CJ, Sneath PH. Constructing a database for low cost identification of Gramnegative rods in clinical laboratories. J Clin Pathol negative rods in

4 Funnell GR, Parkinson DL, Bradbury R. Biochemical identification of Enterobacteriaceae by multipoint inoculation usin

5 Pease AA. Biochemical identification of the Enterobacteriaceae using a multipoint inoculation system. Med Lab

6 Pease AA, Wheat PF, Harris DM. Antimicrobial susceptibility testing and biochemical identification using multipoint inoculation: 5 years' experience. Med Lab Sci 1988;45:28-33.

7 Wheat PF, Pease AA. Identification of Enterobacteriaceae: report of the British Society for Multipoint Technology collaborative study. Med Lab Sci 1989;46:179-85. for identifying Enterobacteriaceae, some Vibrionaceae, identification of Enterobacteriaceae by multipoint Sci 1983;40:349-53. 